\title{
ANÁLISE DA PROFUNDIDADE ÓTICA DE AEROSSÓIS E COEFICIENTE DE ANGSTROM NO CERRADO MATO-GROSSENSE
}

\author{
PALÁCIOS, Rafael da Silva - rafael.pgfa@gmail.com \\ Mestrando em Física Ambiental pela Universidade Federal de Mato Grosso, \\ SALLO, Fernando da Silva - fdss88@gmail.com \\ Doutorando em Física Ambiental pela Universidade Federal de Mato Grosso
}

PRADO, Magdiel Josias do - mgdi.p@hotmail.com

Mestrando em Física Ambiental pela Universidade Federal de Mato Grosso

MUSIS, Carlo Ralph de - carlo.demusis@gmail.com

Doutor e Professor do curso de Pós-Graduação em Física Ambiental pela Universidade Federal de Mato Grosso

NOGUEIRA, José de Souza - nogueira@ufmt.br

Doutor, Professor e coordenador do curso de Pós-Graduação em Física Ambiental pela Universidade Federal de Mato Grosso

\begin{abstract}
RESUMO: Estudos relacionados a aerossóis atmosféricos contribuem para 0 entendimento das propriedades radiativas e seu processo de transmissão à superfície, auxiliando na compreensão dos efeitos diretos e indiretos provocados por eles na atmosfera. Uma das formas de se avaliar os efeitos provocados pelos aerossóis é através da análise de suas propriedades óticas. Desta forma o presente trabalho desenvolve uma análise da Profundidade Ótica de Aerossóis (AOD) e do coeficiente de Angstrom( $\alpha$ ) para uma região de Cerrado no Estado de Mato Grosso. Os dados adquiridos junto a AERONET (AerosolRobotic Network) foram analisados e correlacionados com variáveis meteorológicas de Precipitação, Temperatura de superfície e Umidade Relativa. A AOD total para o comprimento de onda de $500 \mathrm{~nm}$ e suas respectivas parcelas devido à fração fina e grossa de material particulado foi correlacionada, concluindo-se que a AOD nessa região possui uma alta correlação com a fração a moda fina de aerossóis, apresentando um coeficiente de correlação de Spearman de 0,975 com um intervalo de confiança na faixa de 0,996 a 0,981. Os valores obtidos para o coeficiente de Angstrom ( $\alpha$ ) indicaram grande relação do aumento da AOD com as partículas emitidas por queima de biomassa.
\end{abstract}

Palavras - chave: Rede AERONET, queima de biomassa.

DEPTH ANALYSIS OF AEROSOL OPTICAL AND ANGSTROM COEFFICIENT IN SAVANNAH OF MATO GROSSO - BRAZIL

ABSTRACT: Studies related to atmospheric aerosols contribute to the understanding of the radiative properties and the process of transmission to the surface, aiding the comprehension of direct and indirect caused by them in the atmosphere effects. One of the ways to assess the effects caused by aerosols is by analyzing their optical properties. Thus this paper provides an analysis of Aerosol Optical Depth (OD) and the Angstrom coefficient $(\alpha)$ to a region of Cerrado in Mato Grosso. The data acquired from AERONET (Aerosol Robotic Network) were analyzed and correlated with meteorological variables precipitation, surface temperature and Relative Humidity. The total ODA to the wavelength of $500 \mathrm{~nm}$ and their plots due to the fine and coarse fraction of particulate matter was correlated, concluding that the AOD in this region has a high correlation with the fine fraction of aerosols fashion, with a coefficient Spearman correlation of 0.975 with 
a confidence interval ranging from 0.996 to 0.981 . The values obtained for the Angstrom coefficient $(\alpha)$ showed a large increase in the ratio of ODA to the particles emitted by biomass burning.

Keywords: AERONET Network, biomass burning.

\section{INTRODUÇÃO}

Estudos das características de aerossóis em escalas locais e globais, assim como as mudanças dos parâmetros atmosféricos e suas relações com a radiação solar, são de grande importância para a pesquisa atmosférica (WANG et al., 2010; SIVAKUMAR et al., 2010; CHE et al., 2011, KUMAR et al., 2013). Os aerossóis atmosféricos afetam diretamente a transmissão de radiação a superfície terrestre através dos efeitos de espalhamento e absorção (BEEGUM et al., 2009; ZHANG et al., 2013; LI et al.,2014). Indiretamente atuam como núcleos de condensação modificando as propriedades das nuvens e alterando a estabilidade atmosférica (BEEGUM et al., 2009), desempenhando assim um papel significativo no balanço de radiação global, provocam grandes incertezas com relação aos seus efeitos, limitando consideravelmente 0 entendimento do sistema climático global (IPCC, 2007).

Uma das formas de se avaliar os efeitos provocados pelos aerossóis é através da análise de suas propriedades óticas, entre elas a Profundidade de Ótica do Aerossol, (AOD) (AerosolOpticalDepth) que é um indicador da quantidade de aerossóis na coluna vertical da atmosfera, sendo assim, um parâmetro fundamental na avaliação do forçamento radiativo e seu impacto sobre o clima (BALAKRISHNAIAH et al., 2011; ZHANG et al., 2013). Já para caracterizar a dependência espectral das partículas de aerossóis o coeficiente de Angstrom é o parâmetro que melhor explica a relação entre o tamanho das partículas e o comprimento de onda da radiação incidente, desta forma, a partir de valores de espessura ótica em diferentes faixas do espectro pode-se inferir o tamanho médio predominante das partículas. Quanto maior o valor encontrado para o coeficiente de Angstrom, maior a dependência espectral, assim, menor a partícula (CACHORRO et al.,1989; REID et al., 1999).

No Brasil, na bacia Amazônica a composição química da atmosfera sofre grandes mudanças na época da seca, devido às emissões de gases traço e partículas de aerossóis provenientes de queimadas de pastagens e floresta, gerando importantes implicações em nível local, regional e global (ARTAXO et al., 2006). Estudos confirmam a Amazônia como um potencial emissor de material particulado biogênico (GRAHAM et al., 2003; ANDREAE, 2007; RIZZO et al., 2010), mas há também a contribuição de partículas emitidas pela queima de biomassa que influenciam fortemente as taxas fotossintéticas que afetam 0 balanço regional de carbono (OLIVEIRA et al., 2007). Sendo também responsáveis por alterações no balanço radiativo da atmosfera (RAMANATHAN et al., 2001).

O Cerrado brasileiro, ecossistema que só perde em extensão para a floresta Amazônica (RIBEIRO et al., 2011), também é responsável por grandes emissões 
de aerossóis devido a queima de biomassa. A extensão desse bioma abrange aproximadamente $2.100 \mathrm{Km}^{2}$, sendo $50 \%$ considerado terra potencialmente arável (BATLE-BAYER et al., 2010). Depois da Mata Atlântica o Cerrado brasileiro é o bioma que mais sofreu impactos antropogênicos, é classificado como um dos biomas mais ameaçados do mundo, apenas 2,2\% é protegido legalmente (RIBEIRO et al., 2011). Nesse sentido o uso da terra influencia expressivamente nas trocas de energia e massa com a atmosfera. (BATLEBAYER et al., 2010). Nas ultimas décadas grande parte da vegetação natural do Cerrado tem sido substituída por áreas destinadas ás atividades agropecuárias (SANO et al., 2002; SANO et al., 2008). Há também os efeitos de queimadas naturais devido à sazonalidade e consequentemente o grande intervalo de tempo em exposição à seca, cujo início é relativamente consistente de ano para ano, geralmente variando apenas por algumas semanas; elas estão concentradas entre os meses de agosto e novembro, com algumas variações regionais (SCHAFER et al., 2008).

As análises de Profundidade Ótica de Aerossóis, juntamente com os valores para o coeficiente de Angstrom no Cerrado Mato-grossense possibilitam uma caracterização das propriedades óticas da atmosfera local e as influencias locais e globais nas alterações climáticas, bem como auxiliam no entendimento a respeito das trocas radiativas com a superfície para regiões com essas características. A relação entre a sazonalidade e focos de queimadas também é bem definida e dessa forma pode-se analisar qual a significância no aumento da espessura ótica dos aerossóis na estação seca. Sendo assim o objetivo geral desse trabalho é analisar a profundidade ótica de aerossóis no Cerrado Matogrossense e obter os valores de coeficiente de Angstrom através de dados obtidos junto à rede AERONET (AerosolRobotic Network), (HOLBEN et al., 1998, 2001).

\section{MATERIAL E MÉTODOS}

A área de coleta dos dados analisados se encontra localizada na Fazenda Miranda, no município de Cuiabá (Figura 1), Capital do Estado de Mato Grosso, situa-se a aproximadamente $20 \mathrm{~km}$ a sudeste da região urbanizada da Capital. (latitude $16^{\circ} \mathrm{S}$, longitude $56^{\circ} \mathrm{W}$ e com altitude de $175 \mathrm{~m}$ acima do nível do mar), local onde se encontra o fotômetro solar CIMEL controlado pela rede AERONET. Os dados desse sítio são coordenados pela USP, Universidade de São Paulo, cujo responsável é o professor e pesquisador Paulo Eduardo Artaxo Netto (http://lattes.cnpq.br/3977660018939385). Os cuidados de manutenção são realizados pelo grupo de pesquisa de Pós-Graduação em Física Ambiental da Universidade Federal de Mato Grosso. 


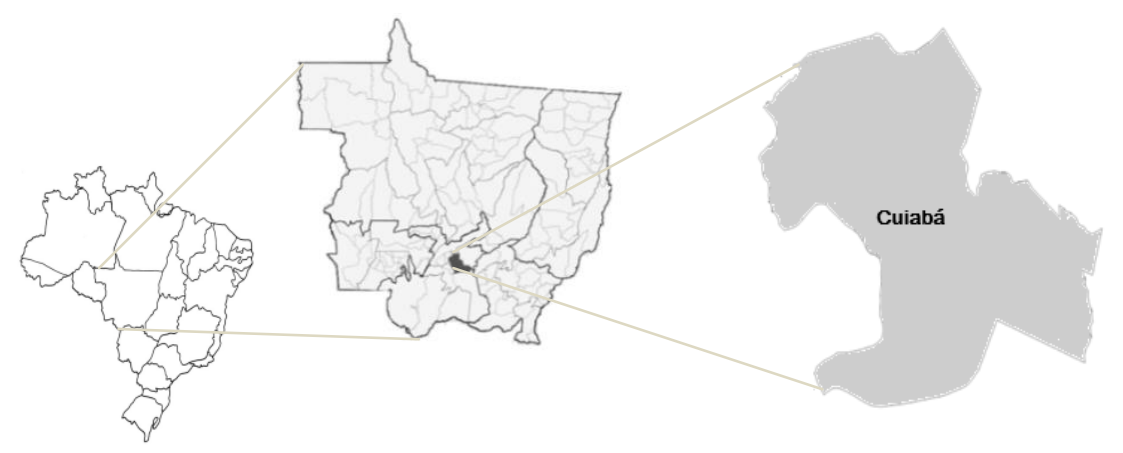

Figura 1 - Localização da área de coleta de dados, no detalhe o município de Cuiabá.

As informações detalhadas sobre a instrumentação, protocolos de medidas, precisão fotométrica, calibração e métodos de processamento podem ser obtidos na obra de Holben et al., (1998 ). Os arquivos de dados da rede AERONET são divididos em três níveis de qualidade: nível 1.0 para os dados brutos, nível 1.5 para dados sem a contaminação de nuvens e nível 2.0 cuja qualidade é certificada pela rede (HOLBEN et al., 1998; SMIRNOV et al., 2000). Os arquivos podem ser baixados do site da AERONET (http://aeronet.gsfc.nasa.gov/). No presente estudo foram utilizados os dados de nível 2.0, tanto para as médias diárias quanto para as mensais para os anos de 2010, 2011 e 2012. Dentre as variáveis disponíveis foi analisado a AOD para a radiação de $500 \mathrm{~nm}$, sendo essa distinta em AOD total, AOD devido à fração fina e AOD devido à fração grossa de aerossóis. Também foi utilizado o coeficiente de Angstrom ( $\alpha$ ) para o intervalo de 440 a $870 \mathrm{~nm}$.

Os dados meteorológicos utilizados nesse trabalho foram adquiridos junto ao Instituto Nacional de Meteorologia (INMET), cuja estação de Cuiabá fica nas coordenadas são $15^{\circ} 61^{\prime} \mathrm{S}$ e $56^{\circ} 10^{\prime} \mathrm{W}$, a aproximadamente $14 \mathrm{~km}$ da posição do fotômetro da rede AERONET, os dados utilizados podem ser acessados através do site http://www.inmet.gov.br/portal/index.php?r=bdmep/bdmep. Foram utilizadas as médias diárias e mensais dos dados de precipitação (PPT), umidade relativa (UR) e temperatura do ar (T) também para os anos de 2010, 2011 e 2012. Também foram utilizados nesse trabalho os dados do Instituto Nacional de Pesquisas Espaciais (INPE), referente ao número de focos de queimadas e incêndios no Estado de Mato Grosso, dados mensais para o período de estudo (2010 a 2012), esses dados estão disponíveis em http://www.inpe.br/queimadas.

O tratamento estatístico dos dados foi realizado com o software IBM SPSS Statistics, versão 20. Uma vez testadas às normalidades das distribuições de dados, foram feitas as análises das relações diárias entre a AOD total, moda fina e moda grossa de aerossóis, juntamente com o coeficiente de Angstrom. Foi feito um teste de correlação, estipulando assim os devidos coeficientes de correlação de Kendall e Spearman, com significâncias de 0,01. A determinação de tais coeficientes foi feito mediante ao processo de Bootstrap com um total de 5000 reamostragens. As medidas diárias de AOD, ainda foram correlacionadas com os dados de Precipitação, Umidade Relativa e Temperatura. Com relação 
aos dados mensais, foi feita uma análise dos registros de focos de queimadas para o Estado de Mato Grosso e sua devida correlação com as médias mensais da AOD total para 500nm.

\section{RESULTADOS E DISCUSSÃO}

A Figura 2 representa a distribuição de frequência dos dados, para os quais foram realizados dois testes de normalidade, Shapiro-Wilk e KolmogorovSmirnov:
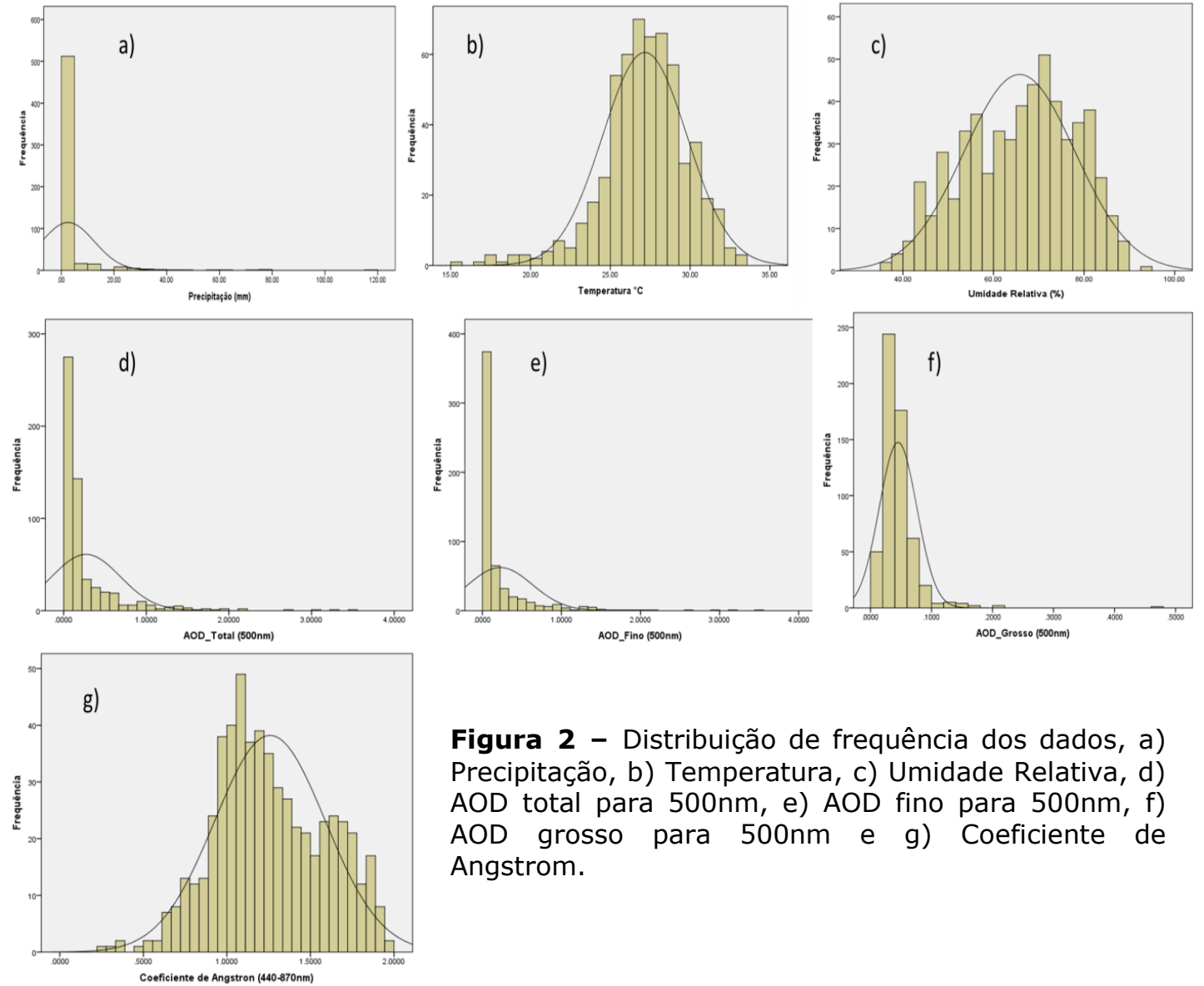

Figura 2 - Distribuição de frequência dos dados, a) Precipitação, b) Temperatura, c) Umidade Relativa, d) AOD total para $500 \mathrm{~nm}$, e) AOD fino para $500 \mathrm{~nm}, \mathrm{f}$ ) AOD grosso para $500 \mathrm{~nm}$ e g) Coeficiente de Angstrom.

Todos dados analisados apresentaram distribuições não normais, comprovadas tanto pelo teste de Shapiro-Wilk como pelo teste de Kolmogorov-Smirnov exceto a distribuição dos dados de Coeficiente de Angstrom no qual foi detectado um p-valor de 0,059 para o teste de Kolmogorov-Smirnov, desta forma foi realizado um terceiro teste, o teste de Lillefors concluído assim pela não normalidade de tais dados. 
A Figura 3 representa as relações entre o total da AOD para o comprimento de onda de 500nm, e suas respectivas parcelas, moda fina e moda grossa. Percebe-se que a grande contribuição para a Profundidade Ótica na área de estudo, é proveniente da parcela de aerossóis de moda fina e que há clara evidencia da sazonalidade no comportamento dos dados.

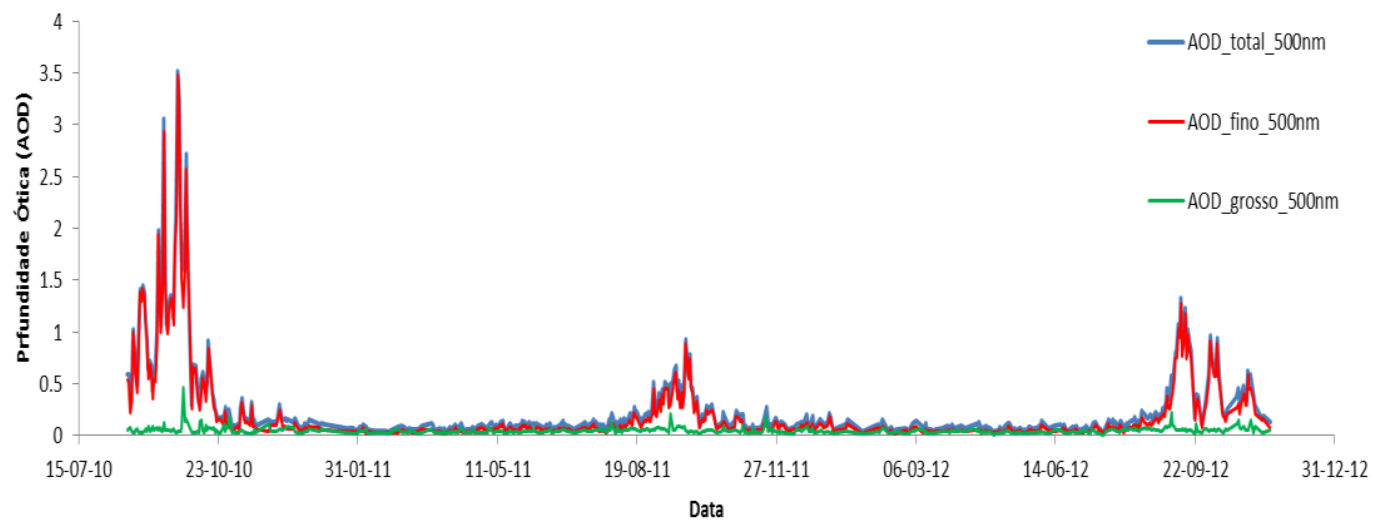

Figura 3 - Distribuição temporal da Profundidade Ótica total e suas parcelas, fina e grossa para o comprimento de onda de $500 \mathrm{~nm}$.

Para condições de atmosfera limpa Houghton et al., (1996) afirma que a Profundidade Ótica é de aproximadamente 0,1 podendo ainda ser inferior a isso. Kumar et al., (2013) em seu trabalho desenvolvido na África do Sul encontrou uma média de 0,3 durante a primavera (setembro-novembro), com o as dados locais (Figura 3) percebe-se que a distribuição apresenta valores abaixo de 0,3 com grandes picos nos meses de agosto e setembro.

Feita a correlação entre o AOD total e suas respectivas parcelas, constatou-se pela Figura 4, o grau de correlação entre a AOD total e sua parcela fina, estatisticamente o coeficiente de correlação de Kendall foi de 0,881 com um intervalo de confiança de 0,863 a 0,897 e o coeficiente de Spearman foi de 0,975 com um intervalo de 0,996 a 0,981. Já para a parcela devido à fração grossa não houve uma correlação estatisticamente significativa. 

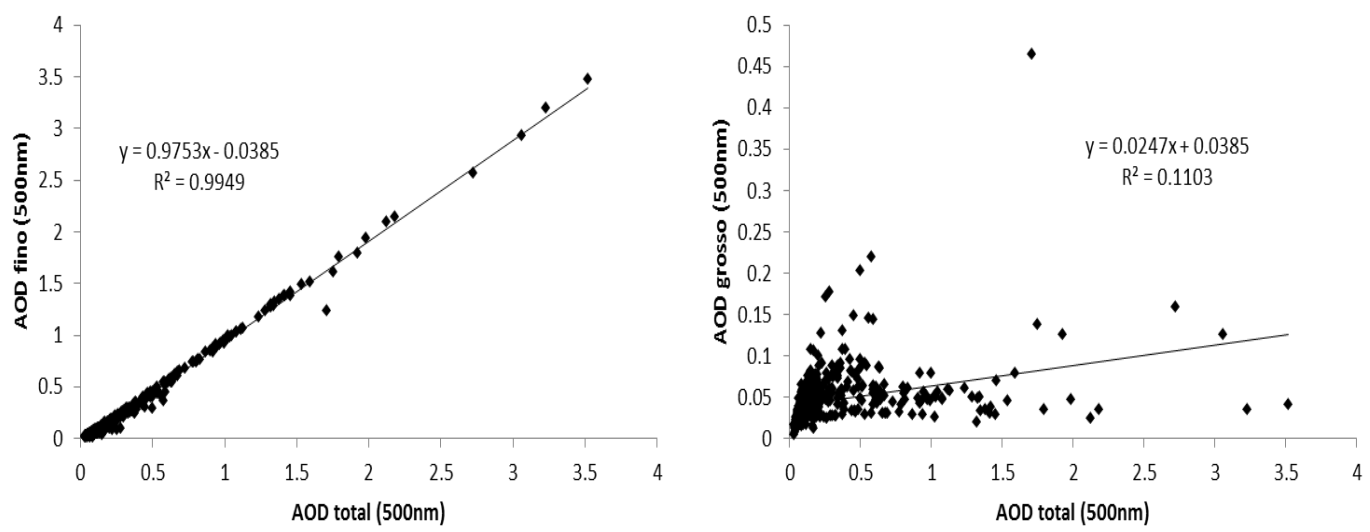

Figura 4 - Correlação entre a AOD total (500nm) e suas parcelas devidas a aerossóis de moda fina e grossa.

A sazonalidade no comportamento dos dados é caracterizada com valores máximos de profundidade óptica na estação seca e mínima na estação chuvosa. Essa variabilidade se deve aos fatores climáticos, econômicos e aos padrões de Precipitação. Verificadas as correlações entre a AOD e as variáveis meteorológicas de Precipitação, Temperatura e Umidade Relativa, não houve uma relação significativa direta, mas através das Figuras 5, 6 e 7 pode-se avaliar a contribuição de cada variável nas variações da AOD.

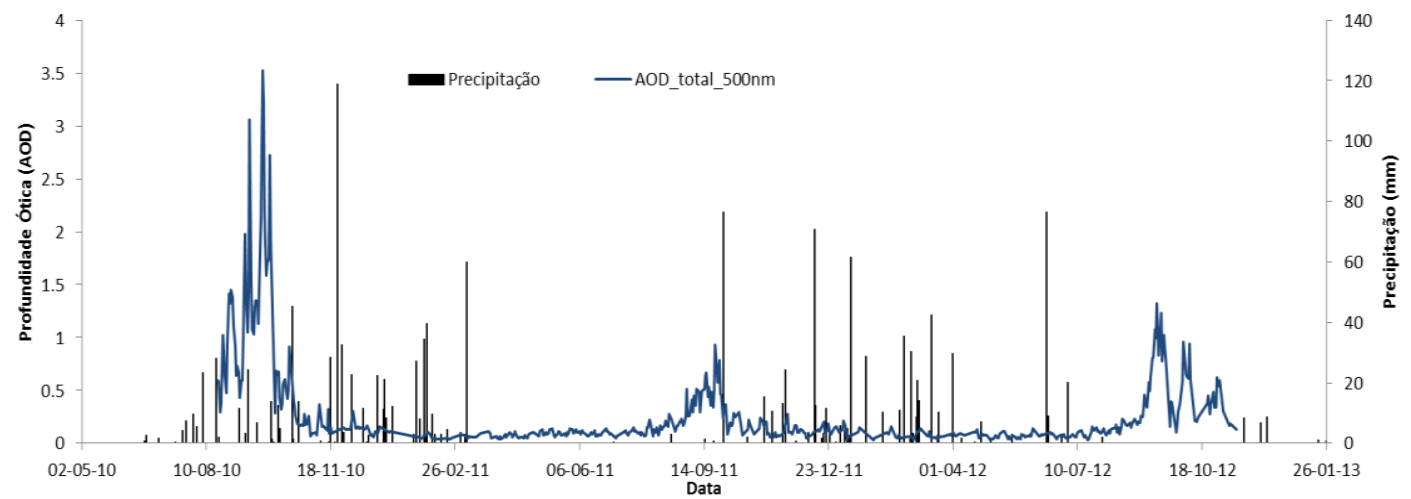

Figura 5 - Distribuição da AOD total (500nm) e Precipitação (mm).

Na Figura 5 percebe-se que os padrões de Precipitação influenciam nos valores da AOD uma vez que quando há a incidência de chuvas a atmosfera é limpa reduzindo assim os valores de Profundida Ótica. Devido à climatologia da região a escassez de Precipitação favorece o aumento do número de focos de queimadas, explicando os altos valores de AOD nos meses de agosto, setembro e outubro. 


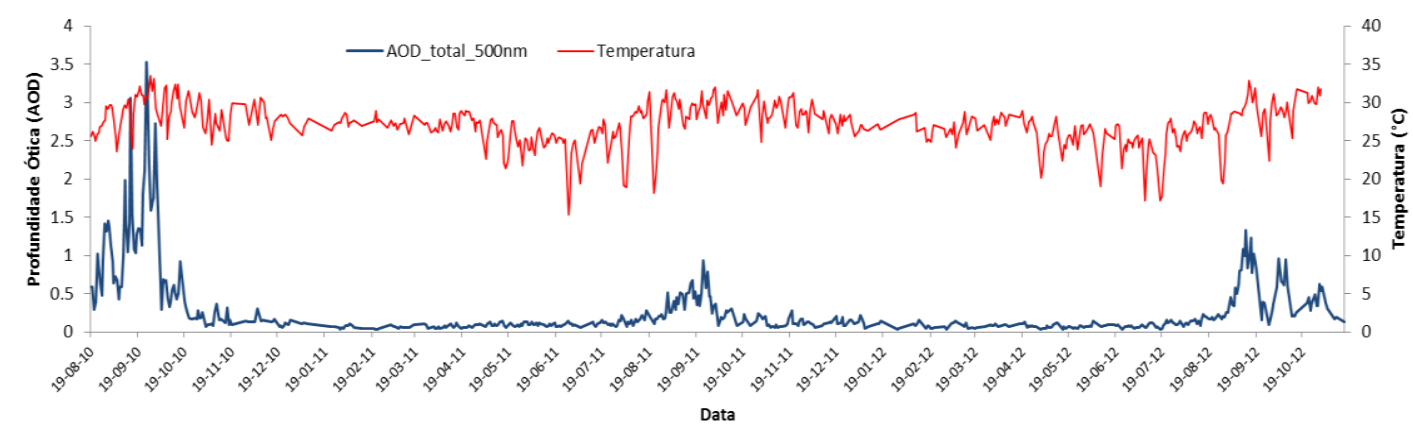

Figura 6 - Distribuição da AOD total (500nm) e Temperatura $\left({ }^{\circ} \mathrm{C}\right)$.

Com relação à Temperatura, a Figura 6 representa as variações das médias diárias ao longo do período estudado, percebe-se que as variações são mais evidentes nos períodos que antecedem as estiagens, estatisticamente não há uma correlação significativa com AOD, mas observa-se uma maior variação da temperatura quando a picos nos valores de Profundidade Ótica.

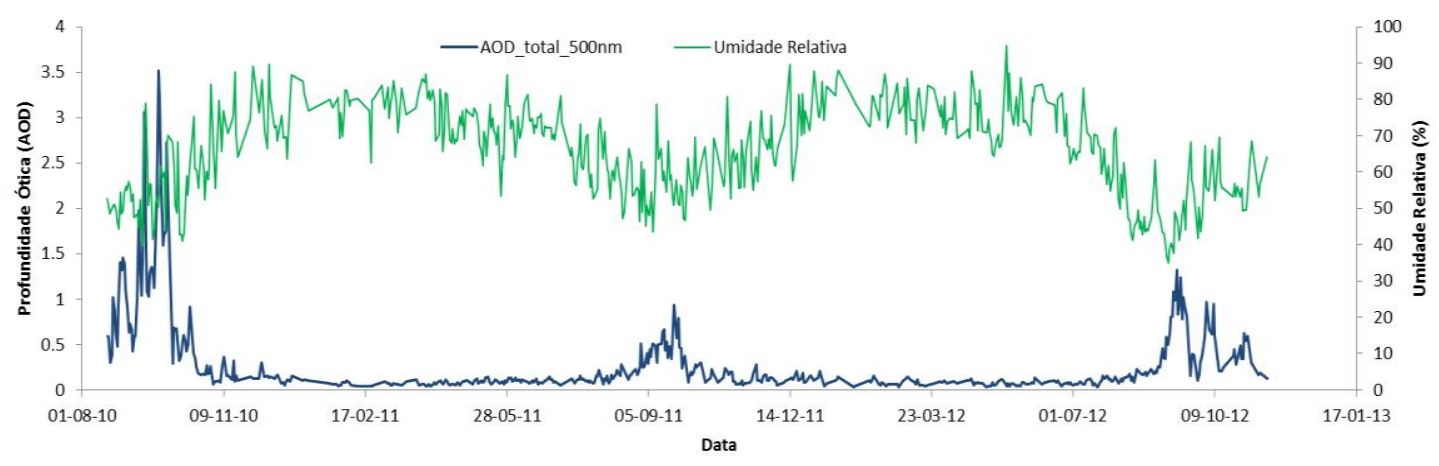

Figura 7 - Distribuição da AOD total (500nm) e Umidade Relativa (\%).

Já a Figura 7, mostra a distribuição da Umidade Relativa, que assim como a Temperatura não apresentou uma correlação estatisticamente significativa, no entanto observa-se, uma queda nos valores de UR quando háaltos valores da $A O D$, fato explicado pelo período de seca no qual a Profundidade ótica tem seus valores máximos.

A variação anual dos picos da AOD é explicada pelo número de registros de focos de queimadas no Estado de Mato Grosso, observa-se (Figura 8) que o maior valor da AOD foi decorrente do mês de setembro de 2010 onde o número de focos de queimadas ultrapassou 18000 registros. 


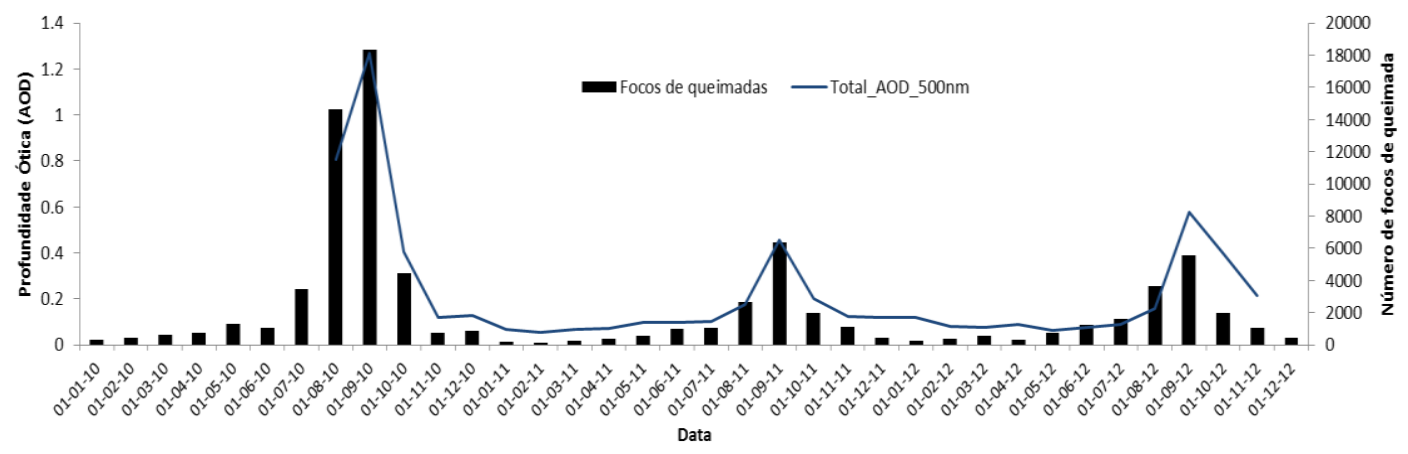

Figura 8 - Distribuição mensal do número de focos de queimada e média mensal da AOD (500nm).

Feita a correlação entre as médias mensais da AOD total para $500 \mathrm{~nm}$ e o número de registros de focos de queimadas obteve-se o coeficiente de correlação de Kendall, 0,653 com um intervalo de confiança de 0,447 a 0,822, já o coeficiente de Spearman foi de 0,829 com um intervalo de 0,601 a 0,938. Considerando que os registros de focos de queimadas são dados mensais para todo o Estado de Mato Grosso a correlação foi significativa, explicando de forma satisfatória as variações da AOD no período de seca.

A variabilidade das concentrações de aerossóis na atmosfera está ligada às condições meteorológicas. Observa-se que nos meses onde há a ocorrência de intensas chuvas, há a remoção do particulado da atmosfera. A chuva também reduz a concentração de poeira emitida pelo solo, reduzindo o material particulado na atmosfera. Já nos meses de junho a outubro onde há estiagem com redução na precipitação, os focos de queimadas atuam consideravelmente no aumento da AOD.

A dependência espectral das propriedades ópticas de aerossóis está relacionada diretamente a distribuição de tamanho do particulado. Sendo assim possível utilizar o coeficiente de Angstrom para se obter informações a respeito do tamanho das partículas em suspensão na atmosfera. Na Figura 9 observa-se a distribuição diária dos valores de coeficiente de Angstrom no intervalo de 440 a $870 \mathrm{~nm}$ e AOD total para 500nm.

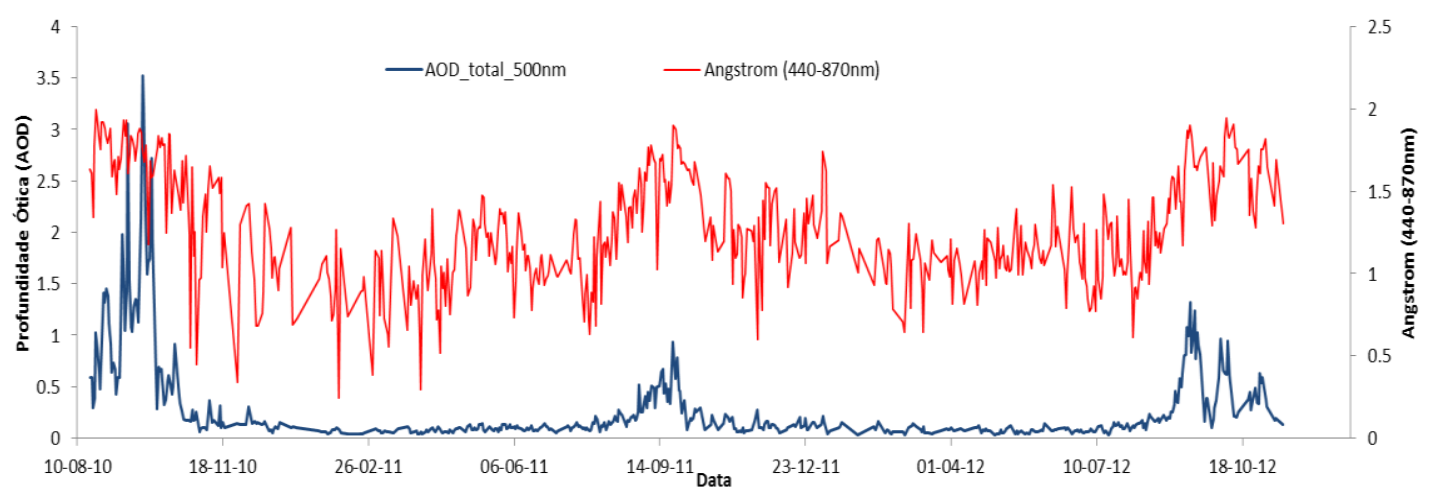


Percebe-se que o aumento da $A O D$, já relacionado com o aumento do número de registros de focos de queimadas na região, contribui diretamente para o aumento da profundidade óptica da parcela das partículas finas (HOLBEN et al., 1996; DUBOVIK et al., 2002; MAGI et al., 2009).Grandes valores de a para o intervalo de $440-870 \mathrm{~nm}$ indicam altas concentrações para partículas finase baixas para partículas grossas. Espera-se que a para 440-870nm seja da ordem de 4 para as partículas de aerossóis muito pequenas (dimensões de moléculas presentes no ar) e se aproxime de 0 para partículas muito grandes (HOLBEN et al., 2001).Observa-se ainda na Figura 9, que o valor de $\alpha$ atingiu extremo superior de 2,0 para os picos da AOD caracterizando o material particulado relativamente pequeno para esses períodos, evidentemente material proveniente da queima de biomassa (ECK et al., 2003b; OGUNJOBI et al., 2004; QUEFACE et al., 2011). Já nos períodos fora da estiagem os valores para $\alpha$ variaram de 0,2 a 1,8 caracterizando uma atmosfera praticamente limpa para estes períodos (ARTAXO et al., 2006).

\section{CONCLUSÕES}

O estudo das propriedades óticas de aerossóis atmosféricos, que no caso desse trabalho foram a Profundidade Ótica (AOD) e coeficiente de Angstrom ( $\alpha$ ), no Cerrado Mato-grossense contribui no entendimento dos processos de transferências radiativas e consequentemente nos impactos diretos e indiretos no clima da região local. Foi feita a análise de correlação entre a AOD total para o comprimento de onda de $500 \mathrm{~nm}$ e suas respectivas parcelas devido à fração fina e grossa de material particulado, concluindo-se que a AOD nessa região possui uma alta correlação com a fração a moda fina de aerossóis apresentando um coeficiente de correlação de Spearman de 0,975 com um intervalo de confiança na faixa de 0,996 a 0,981.

Foi verificado também que não existe uma correlação significativa direta da AOD com as variáveis meteorológicas de Precipitação, Temperatura e Umidade Relativa, no entanto as variações diárias dessas variáveis contribuem para as variações da AOD, principalmente a Precipitação. As características climatológicas locais favoreceram através das estiagens o aumento dos registros de focos de queimadas no Estado de Mato Grosso diretamente correlacionado ao aumento da AOD.

Os valores encontrados para $\alpha$ indicam que a grande contribuição no aumento da AOD nessa região de Cerrado está relacionada à queima de biomassa atingindo valores de 2,0 para os picos nos valores de Profundidade Ótica e números de focos de queimadas. Já para períodos sem grandes estiagens a variou de 0,2 a 1,8 indicando uma atmosfera praticamente limpa.

\section{AGRADECIMENTOS}

Agradecemos a CAPES, pelo apoio financeiro, ao grupo de pesquisa do Programa de Pós-graduação em Física Ambiental e ao Grupo de Estudos do Instituto de Física da USP pela utilização dos dados da rede AERONET.

\section{REFERÊNCIAS}

ANDREAE, M.O. Aerosols before pollution.Science 315, 50e51, 2007. 
ARTAXO P.; OLIVEIRA P. H.; LARA L. L., PAULIQUEVIS T. M.; RIZZO L. V.; PIRES JUNIOR C.; PAIXÃO M. A.; LONGO K. M.; FREITAS S.; CORREIA A. L.. Efeitos climáticos de partículas de aerossóis biogênicos e emitidos em queimadas na Amazônia. Revista Brasileira de Meteorologia, v. 21, n. 3a, p. $168-22,2006$.

BALAKRISHNAIAH G., KUMAR K. R., KUMAR REDDY B. S. K., GOPAL K. R., REDDY R.R., REDDY L.S.S., AHAMMED Y. N., NARASIMHULU K., MOORTHY K. K., BABU S. S. Analysis of optical properties of atmospheric aerosols inferred from spectral AODs and Angström wavelength exponent. Atmospheric Environment 45, 1275-1285, 2011,

BATLLE-BAYER, L.; BATJES, N.H; BINDRABAN, P.S. Changes in organic carbon stocks upon land use conversion in the Brazilian Cerrado: A review. Agriculture, Ecosystems and Environment, v.137, p. 47-58, 2010.

BEEGUM S. N., MOORTHY K. K., BABU S. S. Aerosol microphysics over a tropical coastal station inferred from the spectral dependence of Angstrom wavelength exponent and inversion of spectral aerosol optical depths. Journal of Atmospheric and Solar-Terrestrial Physics 71, 1846-1857, 2009.

CACHORRO V. E., GONZALES M. J., FRUTOS A. M., CASANOVA J. L. Fitting Angstrom's Formulat Spectrally Resolved Aerosol OPTICALTHICKNESS. Atmospheric Environment Vol. 23, No. 1, pp. 265-270. Printed in Great Britain, 1989.

CHE H, WANG Y, SUN J. Aerosol optical properties at Mt. Waliguan Observatory, China. Atmospheric Environment; 45:6004-9, 2011.

DUBOVIK, O.; HOLBEN B. N.; KING M. D.; SMIRNOV A.; ECK T. F.; KINNE S.; SLUTSKER, I. A flexible inversion algorithm for retrieval of aerosol optical properties from sun and sky radiance measurements. Cent.Natl.d'EtudesSpatiales, Meribel, France, v. 99, p. 18-22, 1999.

ECK T. F., HOLBEN B. N., REID J. S., O'NEILL N. T., SCHAFER J. S. , DUBROVNIK O., et al. High aerosol optical depth biomass burning events: a comparison of optical properties for different source regions. Geophys Res Lett, 2003b.

GRAHAM, B., GUYON, P., MAENHAUT, W., TAYLOR, P.E., EBERT, M., MATTHIASMASER, S., MAYOL- BRACERO, O.L., GODOI, R., ARTAXO, P., MEIXNER, F.X., MOURA, M.A., ROCHA, C.H., GRIEKEN, R.V., GLOVSKY, M., FLAGAN, R., ANDREAE, M.O. Composition and diurnal variability of the natural Amazonian aerosol. Journal of Geophysical Research 108 (D24), 4765,2003.

HOLBEN B. N., TANRE D., SMIRNOV A., ECK TF, et al., 2001. An emerging ground-based aerosol climatology: aerosol optical depth from AERONET. Journal of Geophysical Research; 106:12067-97, 2001. 


\section{ISSN : 1980-055x (Impressa) 2237-8642 (Eletrônica)}

HOLBEN, B.N. et al., 1996, Effect of Dry Season Biomass Burning on Amazon Basin Aerosol Concentrations and Optical Properties, 1992-1994. Journal of Geophysical Research, v. 101, p. 19465-19481, 1996.

HOLBEN, B.N., ECK, T.F., SLUTSKER, I., TANRÉ, D., BUIS, J.P., SETZER, A., VERMOTE, E., REAGAN, J.A., KAUFMAN, Y.J., NAKAJIMA, T., LAVENU, F., JANKOWIAK, I., SMIRNOV A. AERONET - a federated instrument network and data archive for aerosol characterization. Remote Sensing of the Environment v.66, n.1, p1-16, 1998.

Houghton, J. T.; MeiraFilho, L. G.; Callander, B. A.; Harrris, N.; Kattenberg, A.; Maskell, K. (Eds) Climate Change 1995: The Science of Climate Change. Cambridge University Press, Cambridge, UK, 1996.

INPE - Instituto Nacional de Pesquisas Espaciais, 2012. Portal do Monitoramento de Queimadas e Incêndios. Disponível em http://www.inpe.br/queimadas. Acesso em: 17/02/2014

intergovernmental paNel on Climate change (IPCC).Climate change. The physical sci-ence basis: Contribution of Working Group I to the Fourth Assessment Report of the Intergovernmental Panel on Climate Change; 129 [Chapter 2], 2007.

KUMAR K. R., SIVAKUMAR V., REDDY R.R., GOPAL K. R., ADESINA A. J. Inferring wavelength dependence of $A O D$ and Angström exponent over a subtropical station in South Africa using AERONET data: Influence of meteorology, long-range transport and curvature effect. Science of the Total Environment 461-462, 397-408, 2013.

LI J., HAN Z., ZHANG R. Influence of aerosol hygroscopic growth parameterization on aerosol optical depth and direct radiative forcing over East Asia. Atmospheric Research 140-141, 14-27, 2014.

MAGI B. I., GINOUX P., MING Y., RAMASWAMY V. Evaluation of optical and extraoptical Southern Hemisphere African aerosol properties simulated by a climate model. Journal of Geophysical Research; 114:D14204, 2009.

OGUNJOBI K. O., HE Z., KIM K. W., KIM Y. J. Aerosol optical depth during episodes of Asian dust storms and biomass burning at Kwangju, South Korea. Atmospheric Environment; 38:1313-23, 2004.

OliVEIRA, P.H.F., ARTAXO, P., PIRES, C., LUCCA, S., PROCOPIO, A., HOLBEN, B., SCHAFER, J., CARDOSO, L.F., WOFSY, S.C., ROCHA, H.R.The effect of biomass burning aerosols and clouds on the CO2 flux in Amazonia. Tellus 59B, 338-349, 2007.

QUEFACE A. J., PIKETH S. J., ECK T. F., TSAY S. C., MAVUME A. F. Climatology of aerosol optical properties in Southern Africa. Atmospheric Environment; 45:2910-21, 2011.

RAMANATHAN, V., CRUTZEN, P.J., KIEHL, J.T., ROSENFELD, D. Aerosols, climate, and the hydrological cycle.Science249, 2119-2124, 2001. 
REID J. S., ECK T. F., CHRISTOPHER S. A., HOBBS P. V., HOLBEN B. N. Use of the Angstrom exponent to estimate and variability of optical and physical properties of aging smoke particles of Brazil. Journal of Geophysical Research; 104(D22):27473-89, 1999.

RIBEIRO, S.C.; FEHRMANN, L.; SOARES, C.P.B.; JACOVINE, L.A.G.; KLEINN, C.; GASPAR, R.O. Above-and Belowground Biomass in Brazilian Cerrado. Forest Ecology And Management, v. 262, p. 491-499, 2001.

RIZZO L.V., ARTAXO P., KARL T., GUENTHER A.B., GREENBERG J. Aerosol properties, in-canopy gradients, turbulent fluxes and VOC concentrations at a pristine forest site in Amazonia. Atmospheric Environment 44, 503-511, 2010.

SANO, E.E.; BARCELLOS, A.O.; BEZERRA, H.S. Assessing the spatial distribution of cultivated pastures in the Brazilian savanna. PasturasTropicales, v.22, p.2-15, 2002.

SANO, E.E.; ROSA, R.; BRITO, J.L.S.; FERREIRA, L.G. Mapeamento semidetalhado do uso da terra do Bioma Cerrado. Pesquisa Agropecuária Brasileira, v.43, p.153-156, 2008.

SCHAFER, J.S.; ECK, T.F.; HOLBEN,B.N.; ARTAXO, P.; DUARTE, A. F.Characterization of the optical properties of atmospheric aerosols in Amazônia from long-term AERONET monitoring (1993-1995 and 1999-2006). Journal of Geophysical Research, v.113, 2008.

SIVAKUMAR V, TESFAYE M, ALEMU W, SHARMA A, BOLLIG C, MENGISTU G. Aerosol measure- ments over South Africa using satellite, Sun-photometer and LIDAR. AdvGeosci; 16:253-62, 2010.

SMIRNOV A., HOLBEN B. N., ECK T. F., DUBOVIK O., SLUTSKER I. Cloud screening and quality con- trol algorithms for the AERONET data base. Remote Sensing of the Environment; 73:337-49, 2000.

WANG P, CHE H, ZHANZ X, SONG Q, WANG Y, ZHAN Z, et al., 2010. Aerosol optical properties of regional background atmosphere in Northeast China. Atmospheric Environment; 44:4404-12, 2010.

ZHANG Z., WENIG M., ZHOU W., DIEHL T., CHAN K., WANG L. The contribution of different aerosol sources to the Aerosol Optical Depth in Hong Kong. Atmospheric Environment 83, 145 -154, 2014. 